\title{
Analysis of mechanical behaviors of waterbomb thin-shell structures under quasi-static load
}

\author{
Lijuan Zhao ${ }^{1}$, Zuen Shang ${ }^{1, *}$, Tianyi Zhang ${ }^{1}$, Zhan Liu ${ }^{1}$, Liguo Han ${ }^{1}$, and Chongwang Wang ${ }^{2}$ \\ 1 Collaborative Innovation Center for Coal Mine Robots, School of Mechanical Engineering, Liaoning Tech- \\ nical University, Fuxin 123000, China; zzz2120@126.com (L.Z.); zty878859579@163.com (T.Z.); \\ liuz0207@163.com (Z.L.); hlg2109@163.com (L.H.) \\ 2 International School of Information and Science \& Engineering, Dalian University of Technology, Dalian \\ 116000, China; 2710333538@mail.dlut.edu.cn (C.W.) \\ * Correspondence: shangzuen@gmail.com (Z.S.); Tel.: +86-041-8511-0068; Fax: +86-041-8511-1125
}

\begin{abstract}
Waterbomb structures are origami-inspired deformable structural components used in new types of robots. They have a unique radially deployable ability that enables robots to better adapt to their environment. In this paper, we propose a series of new waterbomb structures with square, rectangle, and parallelogram base units. Through quasi-static axial and radial compression experiments and numerical simulations, we prove that the parallelogram waterbomb structure has a twist displacement mode along the axial direction. Compared with the square waterbomb structure, the proposed optimal design of the parallelogram waterbomb structure reduces the critical axial buckling load-to-weight ratio by $55.4 \%$ and increases the radial stiffness-to-weight ratio by $67.6 \%$. The significant increase in the radial stiffness-to-weight ratio of the waterbomb structure and decrease in the critical axial buckling load-to-weight ratio make the proposed origami pattern attractive for practical robotics applications.
\end{abstract}

Keywords: Waterbomb structure; Origami pattern; Quasi-static load; Critical axial buckling loadto-weight ratio; Radial stiffness-to-weight ratio

\section{Introduction}

The origami pattern of the origami-inspired waterbomb thin-shell structure consists of a repeating pattern of base units, each with a six-crease vertex, that is, a vertex where six creases converge. When the two ends of a waterbomb thin-shell structure are folded along the axial direction, they can be expanded in the radial direction from the center. This origami structure can be used to improve the robot's environmental adaptability. Different from the conventional mechanical structure, waterbomb structures can change their shapes through reversible elastic deformation. Although they are lightweight, they have the characteristics of high specific stiffness, controllable deformation, and simple assembly. Therefore, thin-walled waterbomb structures have found novel applications in soft robot grippers [1], wearable robots [2], variable-diameter wheel drive robots [3,4], bionic robots [5,6], and floating aerial robots [7]. Equipment functions can be effectively improved by combining cutting-edge origami skills with traditional mechanisms [8].

In recent years, mechanical research related to waterbomb thin-shell structures has yielded numerous results. Feng et al. [9] discovered the twist motion of tubular mechanical metamaterials based on waterbomb origami and analyzed the stiffness changes with the continuous twist motion. Ma et al. [10] developed a waterbomb tubular mechanical metamaterial with programmable deformation-related stiffness and shape modulation functions. Gillman et al. [11] studied the folding mode of the waterbomb structure and explored how the interaction between the stretching energy and the folding ability affect the design of a bistable structure. Fonseca et al. [12] studied the nonlinear dynamics of the waterbomb wheel structure and developed a one-degree-of-freedom reduced-order model system to describe the waterbomb wheel. Hanna et al. [13] performed dynamic 
analysis on the waterbomb base and predicted bistable behavior through kinematic and potential energy analyses. Feng et al. [14] combined kinematics and structural analysis to characterize the folding process of the waterbomb structure and theoretically derived the geometric parameter range of rigid origami motion and nonrigid structure deformation. Bowen et al. [15] established a dynamic model of the waterbomb base to simulate the selffolding behavior of the waterbomb base driven by magneto-active elastomers (MAEs). Glugla et al. [16] used two mechanically different single-layer photopolymers to construct a waterbomb base and demonstrated experimentally that the waterbomb base of this molding method has good load-bearing capacity.

As for the research on the stiffness and critical buckling load of thin-shell structures, Filipov et al. [17] introduced a method of connecting rigid foldable origami tubes with a "zipper" method to effectively improve the stiffness of the system. Berger et al. [18] determined the material geometry needed to achieve the Hashin-Shtrikman upper bound of isotropic elastic stiffness. Bertoldi et al. [19] determined the design principles for the reprogrammable stiffness, shape conversion, and other functions that appear in thin-wall mechanical metamaterials. Ma et al. [20,21] created and studied an energy-absorbing origami structure with periodic gradient stiffness based on the Miura-ori folding pattern. Through energy analysis, Zhai et al. [22] created a thin-walled structure that can selectively collapse along two paths with different stiffness. More mechanical metamaterials with special purposes can be designed by using the principle in this work. Martinez et al. [23] increased the stiffness and anisotropy of elastomeric actuators by introducing a folding structure into the actuators. Filipov et al. [24] improved the simplified bar and hinge model, allowing it to provide a realistic representation of the stiffness characteristics and deformed shapes of origami structures. Guo et al. [25] studied the effect of texturing on the critical pressure of hyperelastic tubes under axial load and internal pressure. You et al. [26] proposed a thin-walled energy absorbing device that reduces the initial buckling force by introducing a pre-folded surface to form geometric imperfections. Holmes [27] found that buckling, wrinkling, folding, creasing, and snapping can be combined as a mechanism to give thin-walled mechanical metamaterials the ability to morph from one shape to another. Lee et al. [28] used the imperfection-sensitive characteristics of thinwalled tubes to guide the deformation process, thereby controlling the buckling behavior of thin-walled tubes. Wang et al. [29] analyzed the elastic buckling of thin-walled tubes and other structures based on Eringen's nonlocal elasticity theory and Timoshenko beam theory. Neves et al. [30] proposed a method to introduce the possibility of critical load control into a topology optimization model. Mehrabadi et al. [31] studied the effects of different geometric parameters and different types of estimation effective material properties on the critical mechanical buckling of functionally graded nanocomposite plates. Wu et al. [32] studied the buckling behavior of single-walled carbon nanotubes subjected to combined hydrostatic pressure and axial compression. Panedpojaman et al. [33] proposed a method to calculate the elastic buckling load of the long axis of cellular columns with multiple end openings. Wagner et al. [34] analyzed the buckling characteristics of thin cylindrical shell structures manufactured through different design concepts such as electroplating, machining, and welding under axial compression. In general, these studies have made great contributions to the practical application of origami structures. However, there is still a lack of detailed mechanical analysis of waterbomb origami structures. The critical buckling load of axial compression (critical buckling load, in short) of waterbomb structures is the parameter required for the waterbomb structure to achieve radial deformation, and the radial stiffness when the waterbomb structure fully deploys is the parameter required for it to achieve load-bearing performance.

In this study, we investigate a series of flexible thin shell structures with base units in the shapes of square, rectangle, and parallelogram. We also conduct finite element simulations and quasi-static axial radial compression testing to study the relationships of critical buckling load and radial stiffness with structural parameters [35]. In Section 2, we present the construction of the waterbomb structures and the corresponding structural parameters. In Section 3, we describe the numerical simulation method and 
corresponding experimental verification. In Section 4, we analyze and discuss the results in detail. In Section 5, we present the conclusions.

\section{GEOMETRIC MODELING}

As shown in Figure 1, all the waterbomb structures studied in this paper have 27 base units arranged in a grid three units wide and nine units tall. Figure 1(a) and (b) are the crease diagrams of the waterbomb origami pattern and the end caps on both ends of the waterbomb structure. The solid lines and dotted lines indicate the mountain and valley creases, respectively. The waterbomb structure shown in Figure 1(c) is formed by tessellation.

Figure 1(a), (b), and (c) show waterbomb structures with square base units, where $m$ and $n$ are the number of horizontal and vertical units. A square base unit is $2 l_{a}$ wide, $t_{a}$ is the length of the triangle centerline spliced on both sides of the opened diagram, and $t_{b}$ is the distance from the center of the regular polygon in the end cap to each vertex. Figure $1(\mathrm{~d})$, (e), and (f) show waterbomb structures with rectangle base units. A rectangle base unit is $2 l_{a}$ long and $2 l_{b}$ wide. Figure $1(\mathrm{~g}),(\mathrm{h})$, and (i) show waterbomb structures with parallelogram base units with the tilt angle $\alpha$.

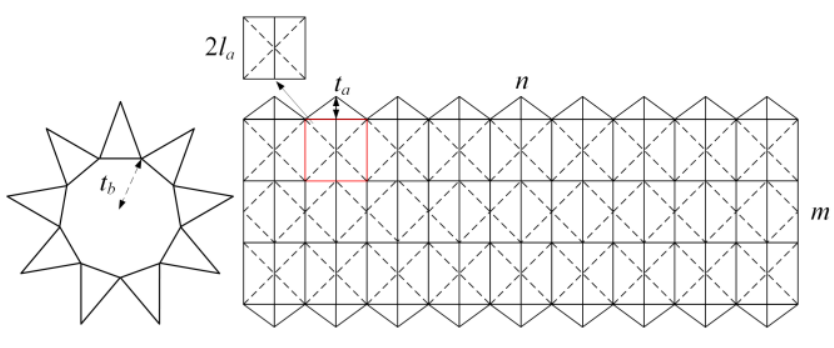

(a)End cap

(b)Crease diagram of square waterbomb structure<smiles></smiles>

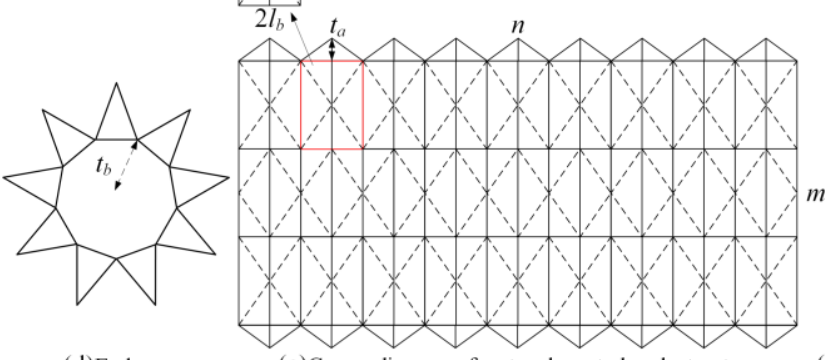

(e)Crease diagram of rectangle waterbomb structure

(d)End cap

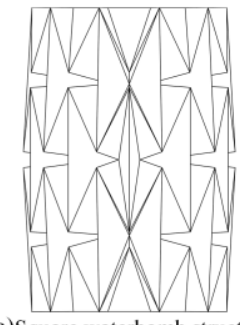

(c)Square waterbomb structure<smiles>C1C2C[As]12</smiles>
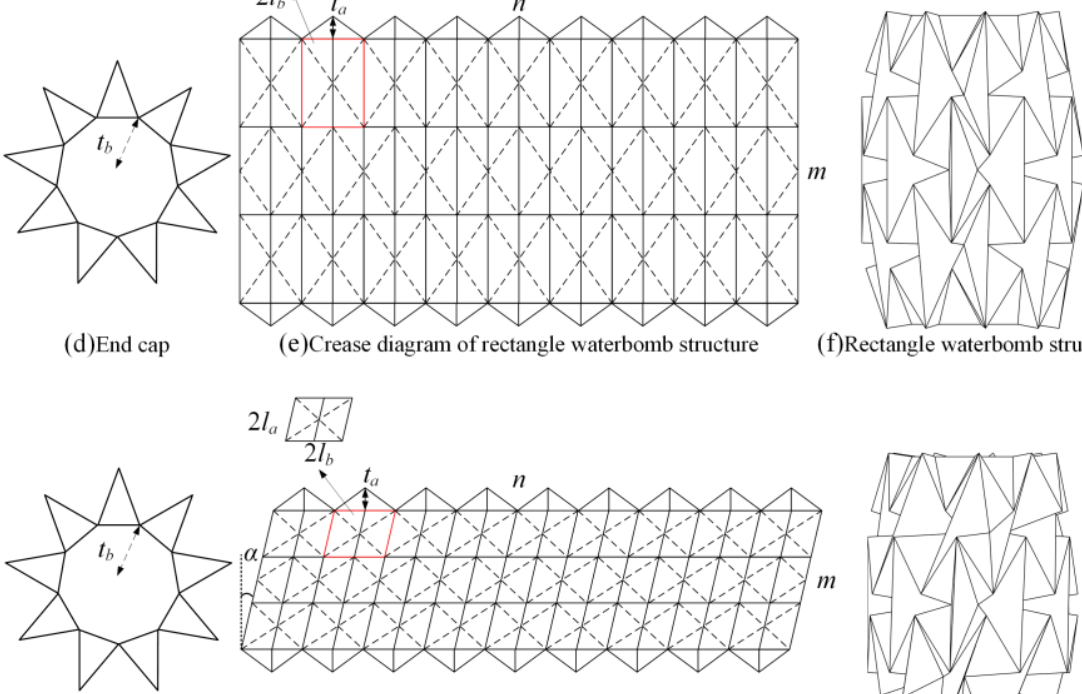

(f)Rectangle waterbomb structure

(g)End cap

(h)Crease diagram of parallelogram waterbomb structure (i)Parallelogram waterbomb structure

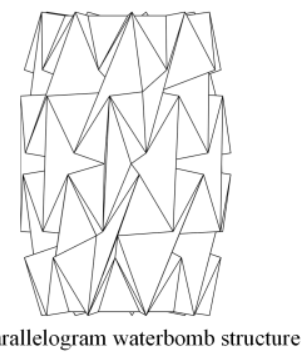

Figure 1. The geometry of waterbomb thin-shell structures: (a) end cap of square waterbomb structure, (b) crease diagram of square waterbomb structure, (c) square waterbomb structure, (d) end cap of rectangle waterbomb structure, (e) crease diagram of rectangle waterbomb structure, (f) rectangle waterbomb structure, (g) end cap of parallelogram waterbomb structure, (h) crease diagram of parallelogram waterbomb structure, and (i) parallelogram waterbomb structure

In addition, $u$ and $v$ are respectively the structure thickness and the gap in the unit facet in the waterbomb structure design. As shown in Figure 2, $v$ describes the amount of soft material used in the waterbomb structure viewed from a side. Table 1 summarizes 
the geometric parameters of waterbomb thin-shell structures formed with the three types of base units.

In total, 34 waterbomb origami structures with different shape parameters are designed in this study, including Z1-Z6 with square base units, J1-J11 with rectangle base units, and P1-P17 with parallelogram base units. For quantitative evaluation, the critical buckling load-to-weight ratio, $Q$, and the radial stiffness-to-weight ratio, $G$, are introduced to facilitate the study of the effects of the parameters. The geometric parameters are listed in Tables 2-4.

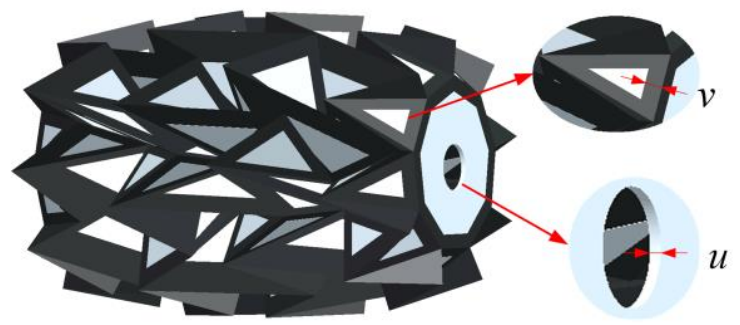

Figure 2. Geometry of the waterbomb thin-shell structure

Table 1. Geometric parameters of the three waterbomb structures

\begin{tabular}{cc}
\hline Type of base units & Geometric parameter \\
\hline Square & $m, n, l_{a}, t_{a}, t_{b}, u, v$ \\
\hline Rectangle & $m, n, l_{a}, l_{b}, t_{a}, t_{b}, u, v$ \\
\hline Parallelogram & $m, n, l_{a}, l_{b}, t_{a}, t_{b}, \alpha, u, v$ \\
\hline
\end{tabular}

Table 2. Structural parameters of square waterbomb

\begin{tabular}{|c|c|c|c|c|c|c|c|c|c|c|c|c|}
\hline Model & $m$ & $n$ & $l_{a}$ & $l_{b}$ & $t_{a}$ & $t_{b}$ & $\alpha$ & $u$ & $v$ & $l_{a} / l_{b}$ & G & $\mathrm{Q}$ \\
\hline & & & $(\mathrm{mm})$ & $(\mathrm{mm})$ & $(\mathrm{mm})$ & $(\mathrm{mm})$ & (rad) & $(\mathrm{mm})$ & $(\mathrm{mm})$ & & $\left(\mathrm{N} / \mathrm{mm} \cdot \mathrm{kg}^{-1}\right)$ & $(\mathrm{N} / \mathrm{kg})$ \\
\hline Z1 & 3 & 9 & 12 & - & 5 & 11.5 & - & 1.5 & 1 & - & 53.6 & 2192.3 \\
\hline $\mathrm{Z} 2$ & 3 & 9 & 12 & - & 5 & 11.5 & - & 1.5 & 1.5 & - & 51.3 & 2076.9 \\
\hline $\mathrm{Z3}$ & 3 & 9 & 12 & - & 5 & 11.5 & - & 1.5 & 2 & - & 49.0 & 2019.6 \\
\hline $\mathrm{Z} 4$ & 3 & 9 & 12 & - & 5 & 11.5 & - & 1.5 & 2.5 & - & 46.5 & 1923.1 \\
\hline Z5 & 3 & 9 & 12 & - & 5 & 11.5 & - & 1.5 & 3 & - & 42.1 & 1781.8 \\
\hline Z6 & 3 & 9 & 12 & - & 5 & 11.5 & - & 1.5 & 3.5 & - & 38.5 & 1750.0 \\
\hline
\end{tabular}

Table 3. Structural parameters of rectangle waterbomb

\begin{tabular}{ccccccccccccc}
\hline Model & $\boldsymbol{m}$ & $\boldsymbol{n}$ & $\boldsymbol{l}_{\boldsymbol{a}}$ & $\boldsymbol{l}_{\boldsymbol{b}}$ & $\boldsymbol{t}_{\boldsymbol{a}}$ & $\boldsymbol{t}_{\boldsymbol{b}}$ & $\boldsymbol{\alpha}$ & $\boldsymbol{u}$ & $\boldsymbol{v}$ & $\boldsymbol{l}_{\boldsymbol{a}} / \boldsymbol{l}_{\boldsymbol{b}}$ & $G$ & \begin{tabular}{c}
$Q$ \\
\hline
\end{tabular} \\
\hline $\mathrm{J} 1$ & 3 & 9 & 10.5 & 16 & 5 & 11.5 & - & 1.5 & 1 & 0.66 & 53.6 & 2192.3 \\
\hline $\mathrm{J} 2$ & 3 & 9 & 11.5 & 15 & 5 & 11.5 & - & 1.5 & 1 & 0.77 & 58.2 & 2019.6 \\
\hline $\mathrm{J} 3$ & 3 & 9 & 13.5 & 13 & 5 & 11.5 & - & 1.5 & 1 & 1.04 & 65.2 & 1727.3 \\
\hline $\mathrm{J} 4$ & 3 & 9 & 14.5 & 12 & 5 & 11.5 & - & 1.5 & 1 & 1.21 & 54.8 & 1614.0 \\
\hline $\mathrm{J} 5$ & 3 & 9 & 15 & 11.5 & 5 & 11.5 & - & 1.5 & 1 & 1.3 & 45.8 & 1607.8 \\
\hline $\mathrm{J} 6$ & 3 & 9 & 16 & 10.5 & 5 & 11.5 & - & 1.5 & 1 & 1.52 & 42.7 & 1365.4 \\
\hline $\mathrm{J} 7$ & 3 & 9 & 13.5 & 13 & 5 & 11.5 & - & 1.5 & 1.5 & 1.04 & 60.6 & 1320.8 \\
\hline $\mathrm{J} 8$ & 3 & 9 & 13.5 & 13 & 5 & 11.5 & - & 1.5 & 2 & 1.04 & 51.2 & 1152.8 \\
\hline $\mathrm{J} 9$ & 3 & 9 & 13.5 & 13 & 5 & 11.5 & - & 1.5 & 2.5 & 1.04 & 49.6 & 1054.5 \\
\hline $\mathrm{J} 10$ & 3 & 9 & 13.5 & 13 & 5 & 11.5 & - & 1.5 & 3 & 1.04 & 44.2 & 962.3 \\
\hline $\mathrm{J} 11$ & 3 & 9 & 13.5 & 13 & 5 & 11.5 & - & 1.5 & 3.5 & 1.04 & 40.7 & 854.5 \\
\hline
\end{tabular}


Table 4. Structural parameters of parallelogram waterbomb

\begin{tabular}{|c|c|c|c|c|c|c|c|c|c|c|c|c|}
\hline Model & $m$ & $n$ & $l_{a}$ & $l_{b}$ & $t_{a}$ & $t_{b}$ & $\alpha$ & $u$ & $v$ & $l_{a} / l_{b}$ & $G$ & $Q$ \\
\hline & & & $(\mathrm{mm})$ & $(\mathrm{mm})$ & $(\mathrm{mm})$ & $(\mathrm{mm})$ & (rad) & $(\mathrm{mm})$ & $(\mathrm{mm})$ & & $\left(\mathrm{N} / \mathrm{mm} \cdot \mathrm{kg}^{-1}\right)$ & $(\mathrm{N} / \mathrm{kg})$ \\
\hline P1 & 3 & 9 & 13.5 & 13.5 & 5 & 11.5 & 0.105 & 1.5 & 1 & 1 & 75.55 & 1519.23 \\
\hline P2 & 3 & 9 & 13.5 & 13.5 & 5 & 11.5 & 0.209 & 1.5 & 1 & 1 & 78.98 & 1442.31 \\
\hline P3 & 3 & 9 & 13.5 & 13.5 & 5 & 11.5 & 0.314 & 1.5 & 1 & 1 & 84.53 & 1365.38 \\
\hline $\mathrm{P} 4$ & 3 & 9 & 13.5 & 13.5 & 5 & 11.5 & 0.419 & 1.5 & 1 & 1 & 74.86 & 1326.92 \\
\hline P5 & 3 & 9 & 13.5 & 13.5 & 5 & 11.5 & 0.524 & 1.5 & 1 & 1 & 68.68 & 1269.23 \\
\hline P6 & 3 & 9 & 13.5 & 13.5 & 5 & 11.5 & 0.628 & 1.5 & 1 & 1 & 65.25 & 1057.69 \\
\hline P7 & 3 & 9 & 13.5 & 13.5 & 5 & 11.5 & 0.314 & 1.5 & 1.5 & 1 & 83.01 & 1132.08 \\
\hline P8 & 3 & 9 & 13.5 & 13.5 & 5 & 11.5 & 0.314 & 1.5 & 2 & 1 & 82.14 & 900.00 \\
\hline P9 & 3 & 9 & 13.5 & 13.5 & 5 & 11.5 & 0.314 & 1.5 & 2.5 & 1 & 76.92 & 826.92 \\
\hline P10 & 3 & 9 & 13.5 & 13.5 & 5 & 11.5 & 0.314 & 1.5 & 3 & 1 & 71.39 & 769.23 \\
\hline P11 & 3 & 9 & 13.5 & 13.5 & 5 & 11.5 & 0.314 & 1.5 & 3.5 & 1 & 65.14 & 730.77 \\
\hline $\mathrm{P} 12$ & 3 & 9 & 10.5 & 16 & 5 & 11.5 & 0.314 & 1.5 & 1 & 0.66 & 61.8 & 1423.1 \\
\hline P13 & 3 & 9 & 11.5 & 15 & 5 & 11.5 & 0.314 & 1.5 & 1 & 0.77 & 58.2 & 1529.4 \\
\hline P14 & 3 & 9 & 13.5 & 13 & 5 & 11.5 & 0.314 & 1.5 & 1 & 1.04 & 62.3 & 1290.9 \\
\hline P15 & 3 & 9 & 14.5 & 12 & 5 & 11.5 & 0.314 & 1.5 & 1 & 1.21 & 60.3 & 1333.3 \\
\hline P16 & 3 & 9 & 15 & 11.5 & 5 & 11.5 & 0.314 & 1.5 & 1 & 1.3 & 50.4 & 1411.8 \\
\hline P17 & 3 & 9 & 16 & 10.5 & 5 & 11.5 & 0.314 & 1.5 & 1 & 1.52 & 56.1 & 1442.3 \\
\hline
\end{tabular}

\section{SIMULATION AND EXPERIMENT}

\subsection{Simulation model}

The explicit dynamics solver of the finite element software ABAQUS/Explicit is used to simulate the axial and radial compression processes of the waterbomb structure with different types of base units and to solve highly nonlinear problems. In order to accurately simulate the large deformation states of the waterbomb structures, S4R shell elements are used. To ensure mesh convergence, the mesh size is set to $2 \mathrm{~mm}$. Considering the surface contact, the static friction coefficient and the dynamic friction coefficient are both set to 0.25 . The types of soft and hard materials are shown in Figure 3, and the material properties are shown in Table 5.

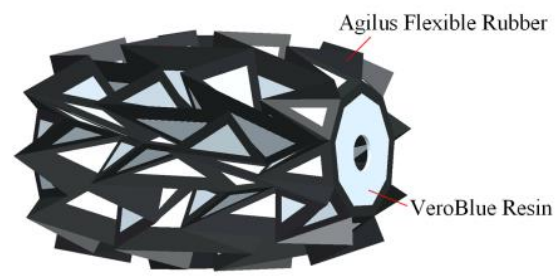

Figure 3. Areas of construction materials

Table 5. Material properties

\begin{tabular}{|c|c|c|c|c|c|c|}
\hline Material & $\begin{array}{c}\text { Elastic } \\
\text { modulus } \\
(\mathrm{MPa})\end{array}$ & $\begin{array}{l}\text { Poisson's } \\
\text { ratio }\end{array}$ & $\begin{array}{c}\text { Tensile } \\
\text { strength } \\
\text { (MPa) }\end{array}$ & $\begin{array}{c}\text { Shear } \\
\text { modulus } \\
(\mathrm{MPa})\end{array}$ & $\begin{array}{l}\text { Polymer } \\
\text { density } \\
\left(\mathrm{g} / \mathrm{cm}^{3}\right)\end{array}$ & $\begin{array}{c}\text { Percentage } \\
\text { elongation at } \\
\text { fracture }(\%)\end{array}$ \\
\hline VeroBule rubber & 2500 & 0.35 & 60 & 926 & $1.18-1.19$ & $15-25$ \\
\hline Agilus resin & $1 \times 1011$ & 0.45 & 2.4 & 0.238 & $1.14-1.15$ & $220-240$ \\
\hline
\end{tabular}


The simulation is carried out in two steps. In the first step, the critical axial buckling load is solved in the following manner. The waterbomb structure is placed between two rigid plates; one rigid plate remains fixed, and the other gradually compresses the sample. The compression displacement is $45 \mathrm{~mm}$. The simulation results are shown in Figure 6. In the second step, the radial stiffness is solved in the following manner. When fully compressed (that is, the waterbomb structure is compressed until the middle units are fully expanded), the rigid plate is set to be tangent to the expanded state of the waterbomb structure, and the type of contact is defined as point-to-surface contact. The sample is compressed upwards perpendicularly in the horizontal direction. The compression displacement is $34.5 \mathrm{~mm}$. The simulation results are shown in Figure 7.

In terms of $Q$ and $G$, which are used as evaluation indicators, 34 waterbomb samples are evaluated. $Q$ and $G$ are defined in Equations (1) and (2), respectively:

$$
\begin{gathered}
Q=\frac{F_{b}}{M}, \\
G=\frac{U}{M}=\frac{F}{M s},
\end{gathered}
$$

where $F_{b}$ is the critical buckling load, $M$ is the mass of the waterbomb structure, $F$ is the maximum radial load, and $s$ is the radial displacement.

\subsection{Experimental verification}

In order to verify the accuracy of the ABAQUS simulation method, the square waterbomb structure $\mathrm{Z3}$, the rectangle waterbomb structure J8, and the parallelogram waterbomb structure P8 are used in the comparative experiment. Quasi-static axial load tests and radial load tests are carried out to verify the validity of the numerical model. The waterbombs are made of soft and hard materials by 3D printing. The soft and hard materials are Agilus soft rubber and VeroBule resin, respectively, which adhere well to each other. The models are in a scale of 1:1. This fabrication process is quick and accurate, and it is convenient because one-time molding does not require complex assembly. Figure 4(a) shows the fabrication process, Figure 4(b) shows the process of removing the support material, and Figure 5 shows a finished sample to be tested.

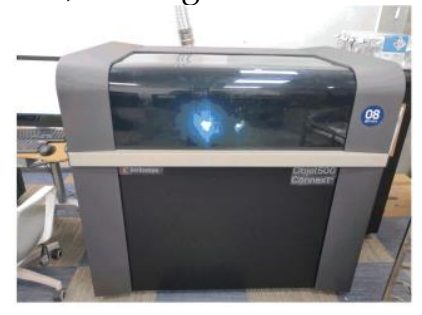

(a)

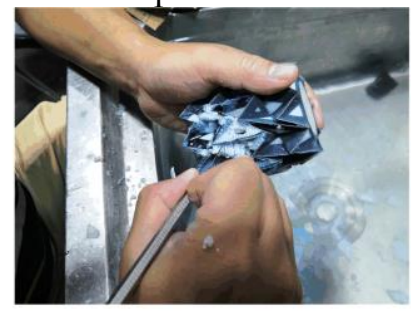

(b)

Figure 4. Waterbomb thin-shell structure fabrication: (a) 3D printing and (b) support material removal

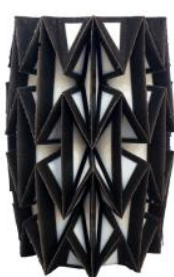

(a)

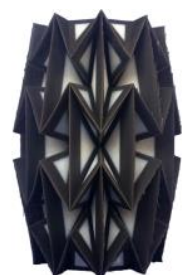

(b)

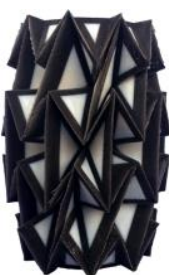

(c)

Figure 5. Waterbomb thin-shell structure: (a) square waterbomb structure test sample Z3, (b) rectangle waterbomb structure test sample J8, and (c) parallelogram waterbomb structure test sample P8

The WDW-10D and WDW-2D electronic universal testers are used to perform quasistatic axial compression tests and radial compression tests on the waterbomb samples. The axial compression test process is shown in Figure 6. During the process, the 

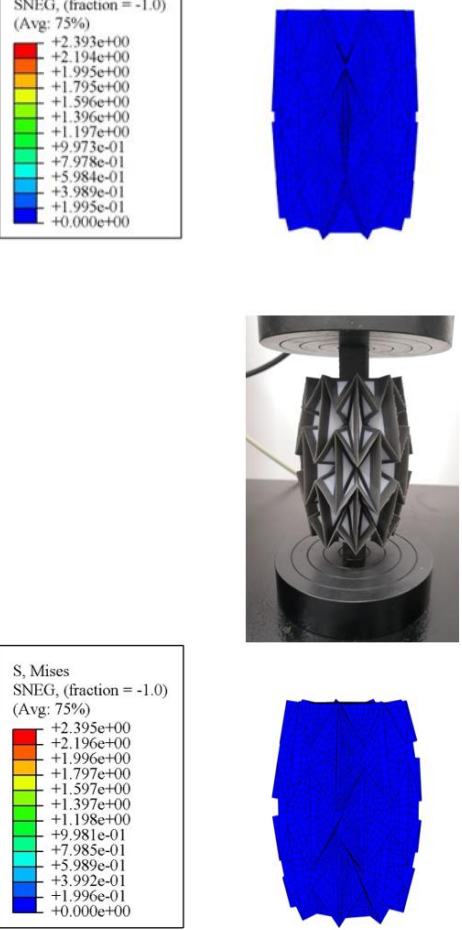

waterbomb structure is placed between two $3 \mathrm{~cm}^{3}$ sample plates, and a compression distance of $45 \mathrm{~mm}$ is applied at a loading rate of $1 \mathrm{~mm} \mathrm{~min}^{-1}$. The entire test process is recorded.

The radial compression test process is shown in Figure 8. During this process, the 3D printing mold is used to define the expansion state of the waterbomb structure, and the waterbomb structure is placed in the mold to make the waterbomb structure fully expand radially. Then a compression distance of $34.5 \mathrm{~mm}$ is applied at a loading rate of $1 \mathrm{~mm}$ $\mathrm{min}^{-1}$. The entire test process is recorded.

Comparisons between the axial test results and the simulation results as well as between the radial test results and the simulation results are shown in Figures 10 and 11, respectively. The calculations show that the maximum errors between the simulation and experimental test results of the square, rectangle, and parallelogram waterbombs are $9.34 \%, 9.88 \%$, and $9.76 \%$, respectively. Therefore, the finite element method is considered suitable for this study. The main reason for these errors is the errors between the numerical model and sample dimensions due to inaccuracy in machining. Because our study focuses on the overall mechanical properties of the waterbomb structures, we do not explore these factors.
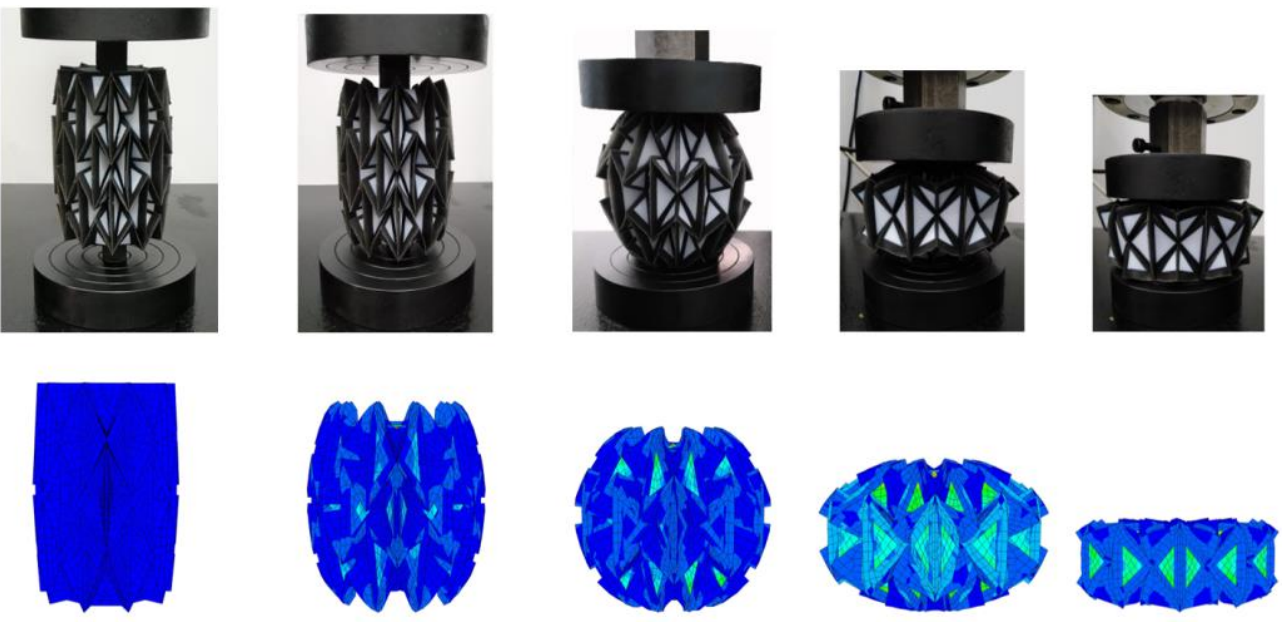

(a)
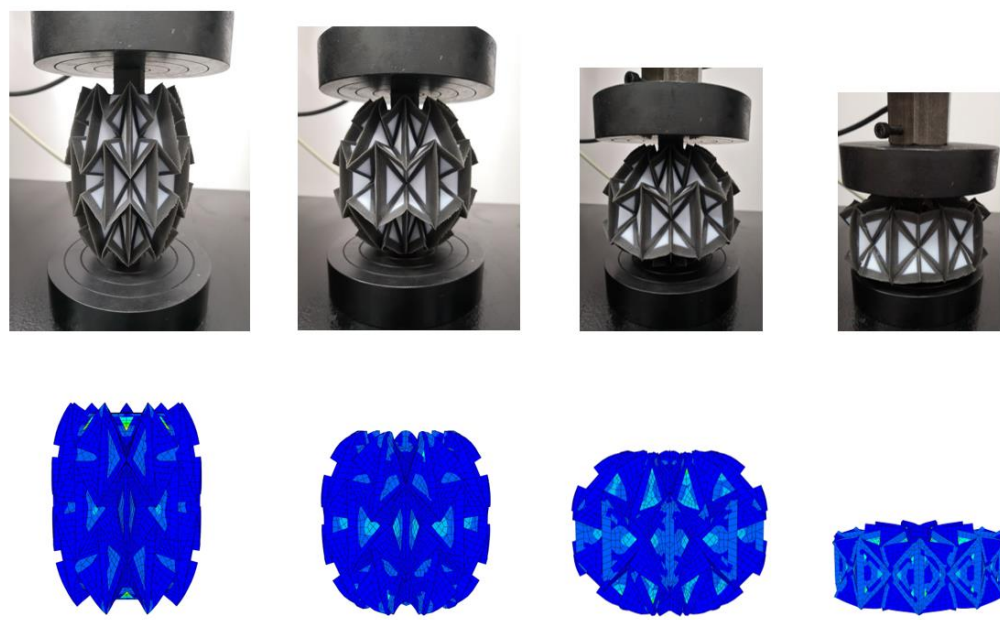

(b) 

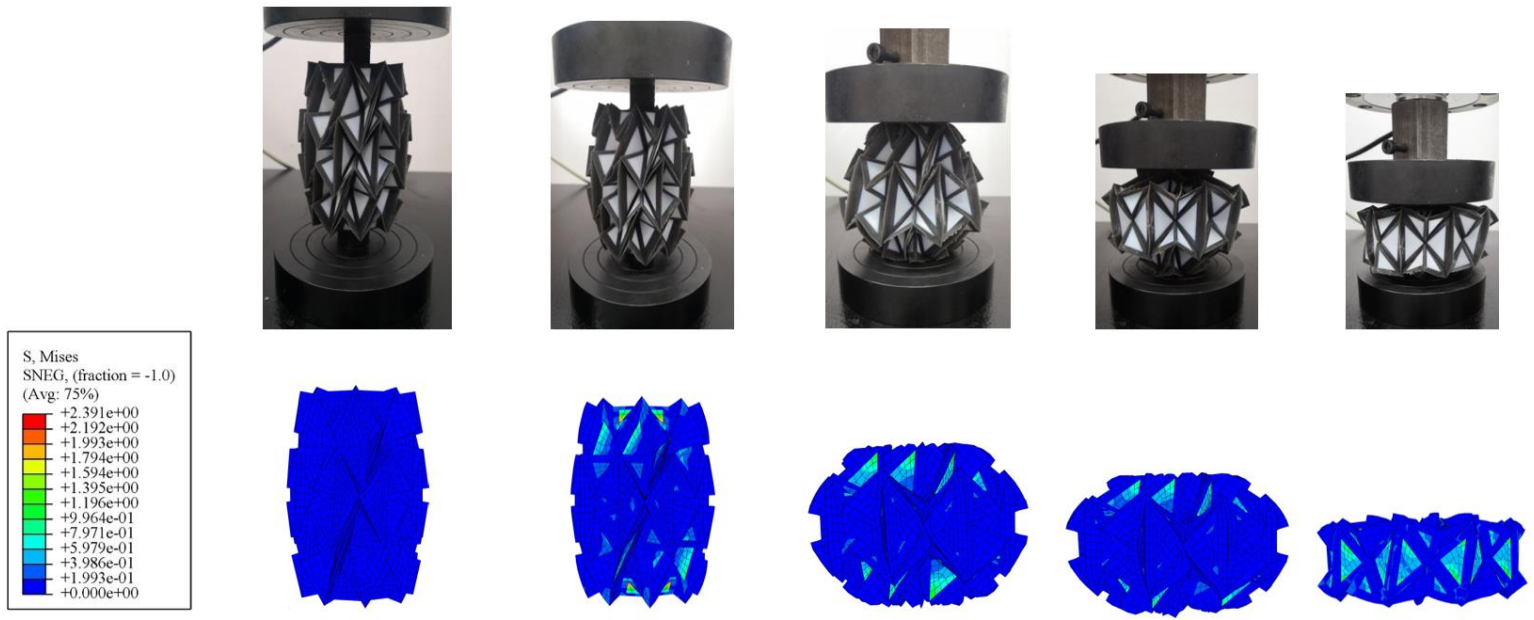

(c)

Figure 6. Axial compression process of waterbomb structure: (a) square waterbomb structure test sample Z3, (b) rectangle waterbomb structure test sample J8, and (c) parallelogram waterbomb structure test sample P8

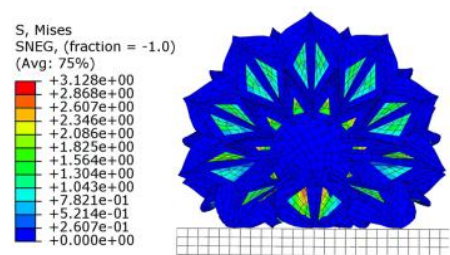

(a)

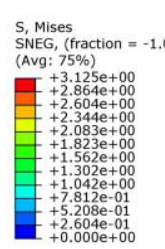

$+2.604 \mathrm{e}-01$
$+0.000 \mathrm{e}+00$

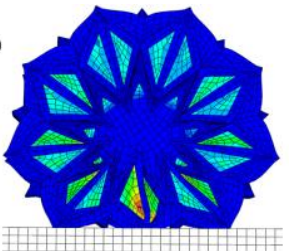

(b)

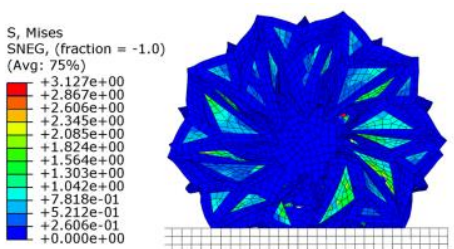

(c)

Figure 7. Radial compression process of waterbomb structure: (a) square waterbomb structure test sample Z3, (b) rectangle waterbomb structure test sample J8, and (c) parallelogram waterbomb structure test sample P8

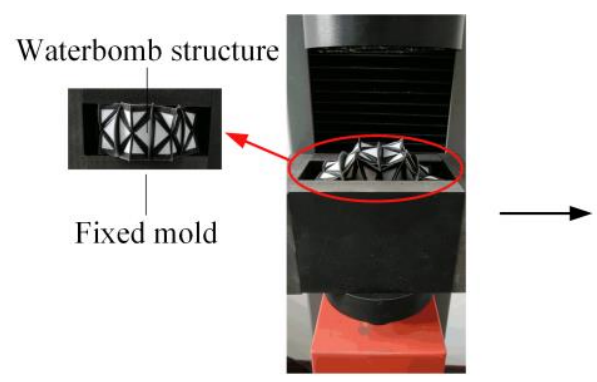

(a)

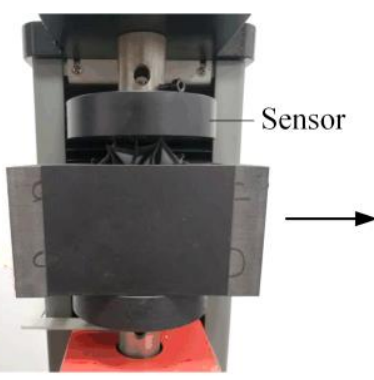

(b)

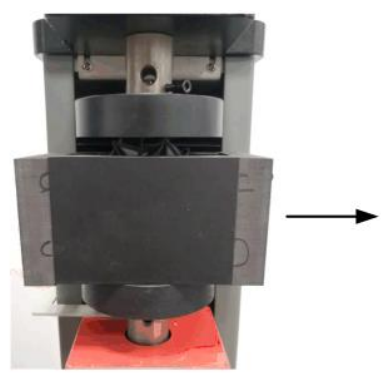

(c)

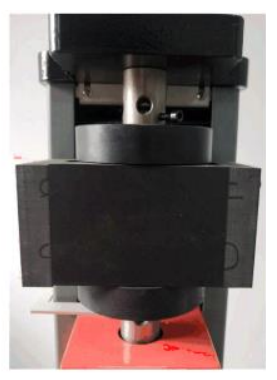

(d)

Figure 8. Experimental process of radial compression of waterbomb structure: (a) the waterbomb structure is mounted, (b) the compression displacement is $0 \mathrm{~mm}$, (c) the compression displacement reaches $17.25 \mathrm{~mm}$, and (d) the compression displacement reaches $34.5 \mathrm{~mm}$ 

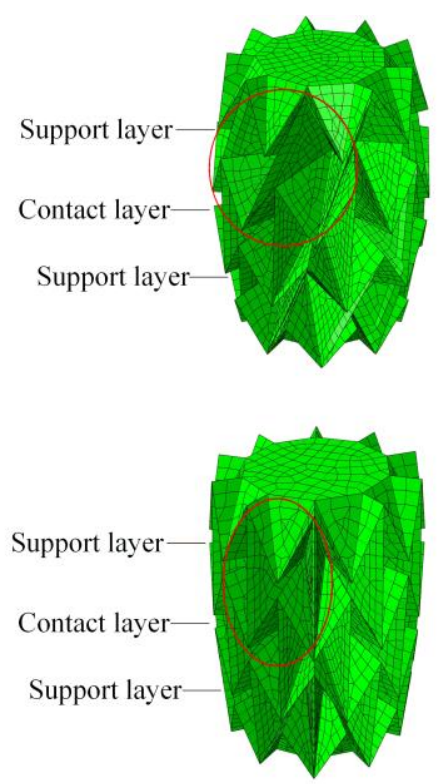
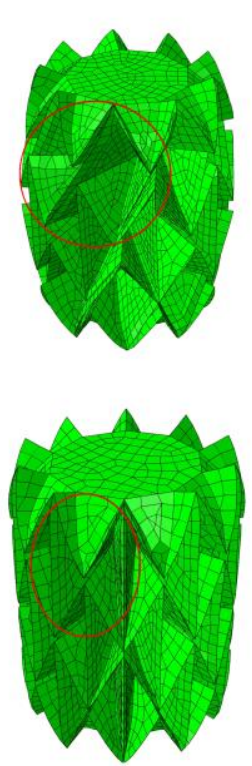

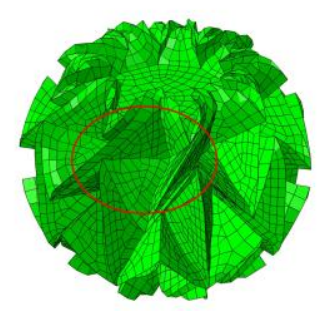

(a)

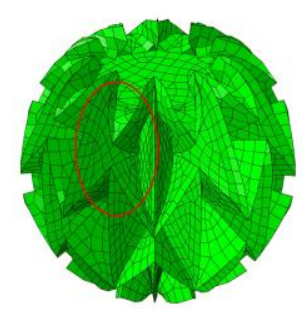

(b)
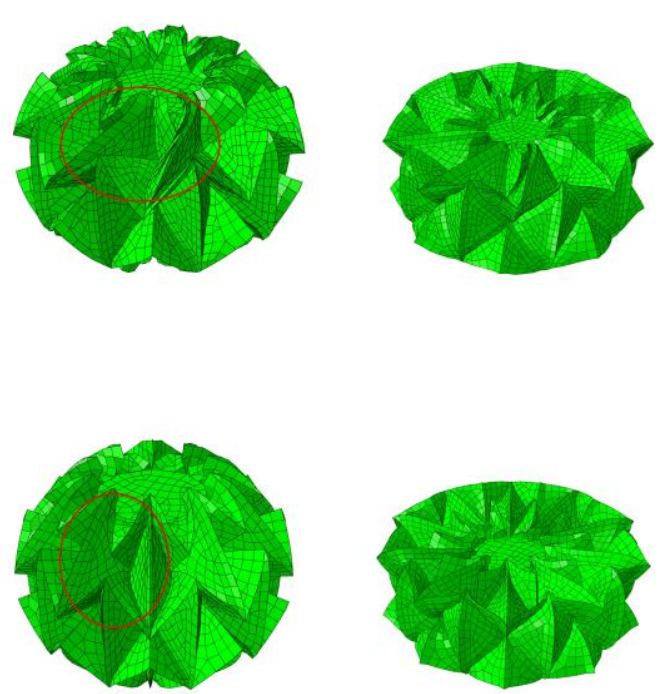

Figure 9. Waterbomb structures: (a) the parallelogram waterbomb structure test sample P8, and (b) the square waterbomb structure test sample Z3

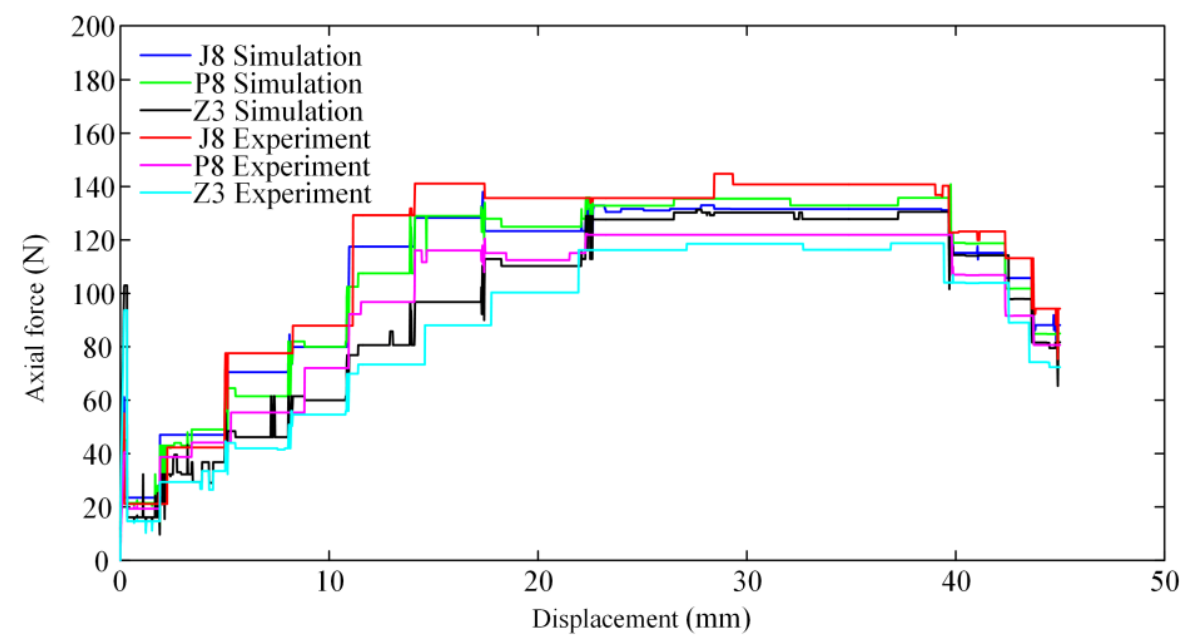

Figure 10. Experimental and numerical axial force-displacement curves of the three waterbomb structures

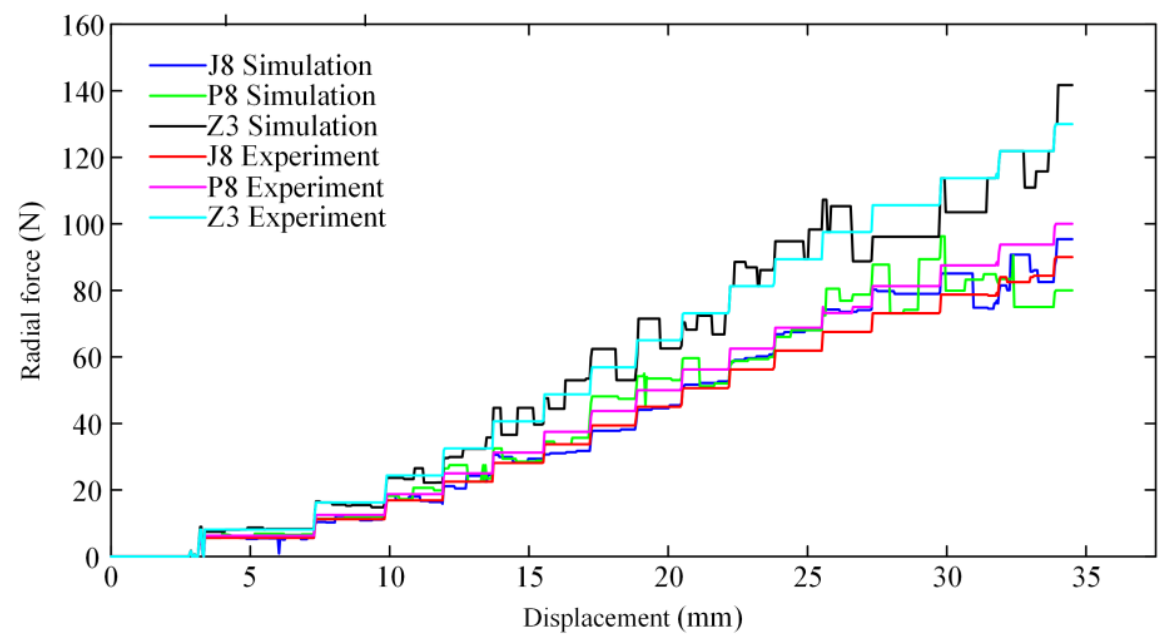

Figure 11. Experimental and numerical radial force-displacement curves of the three waterbomb structures 


\section{RESULTS AND DISCUSSION}

\subsection{Comparison of the square waterbomb structure and the parallelogram waterbomb structure}

We first compare the parallelogram waterbomb structure P8 and the square waterbomb structure $\mathrm{Z3}$. The axial compression process and the radial compression process of $\mathrm{Z} 3$ are shown in Figure 6(a) and Figure 7(a), respectively. The axial compression process and the radial compression process of P8 are shown in Figure 6(c) and Figure 7(c), respectively. The parallelogram waterbomb structure changes with the compression displacement, which triggers the axial twist displacement mode. It can be seen from Figure 9(a) that the twist displacement mode occurs in the support layers of the parallelogram waterbomb structure. As shown in Figure 9(b), the square waterbomb structure changes with the compression displacement, and the overall structure gradually expands radially outward with creases along the axial direction.

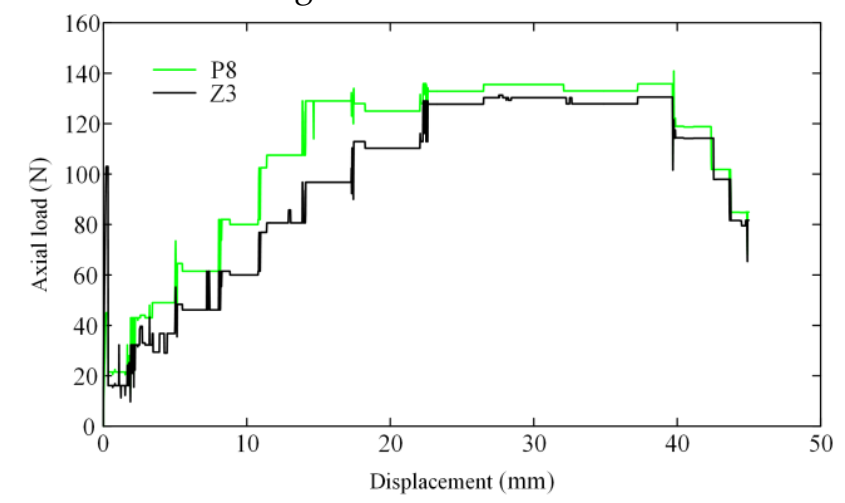

Figure 12. Axial load-displacement curves

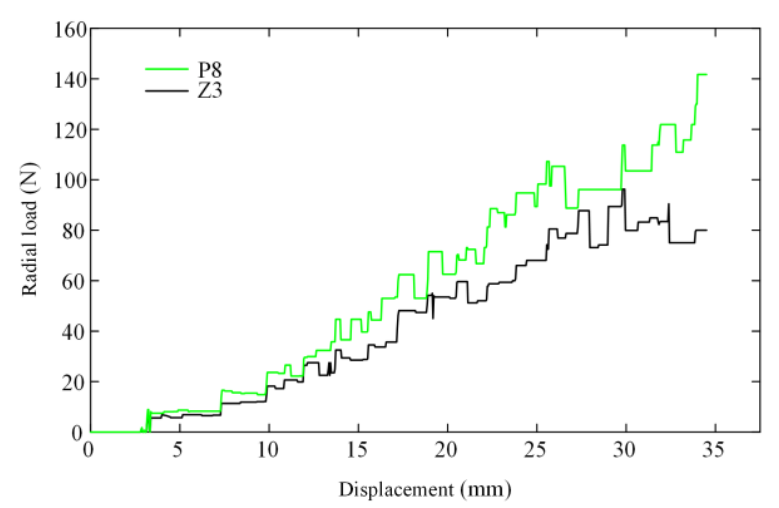

Figure 13. Radial load-displacement curves

The force-displacement curves of the two models are shown in Figures 12 and 13. The numerical data are shown in Tables 2 and 4. Compared with Z3, the critical buckling load-to-weight ratio of $\mathrm{P} 8$ is reduced by $55.4 \%$, and the radial stiffness-to-weight ratio of P8 is increased by $67.6 \%$. This is because the axial stiffness of the overall structure is reduced when the parameters of the parallelogram waterbomb structure are adjusted. The radial stiffness of the structure is improved in its deployed state.

\subsection{Effect of side ratio}

The axial compression process of $\mathrm{J} 8$ is shown in Figure 6. The radial compression process of $\mathrm{J} 8$ is shown in Figure 7(b). In order to analyze the effects of the side ratio, six types of rectangle waterbomb structure models (J1-J6) with different side ratios are established and analyzed. The specific parameters are shown in Table 3. J1-J6 are simulated, and the numerical calculation results yield the trends of the critical buckling load-toweight ratio and the radial stiffness-to-weight ratio with respect to the side ratio as shown in Figures 14 and 15, respectively. 


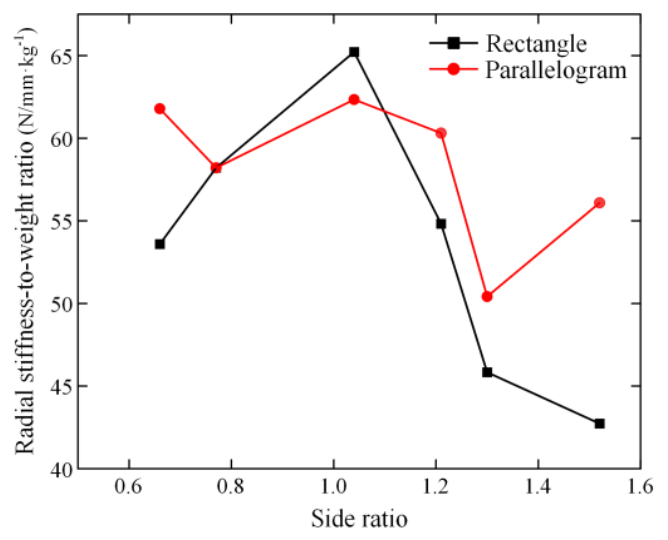

Figure 14. Radial stiffness-to-weight ratio vs. side ratio

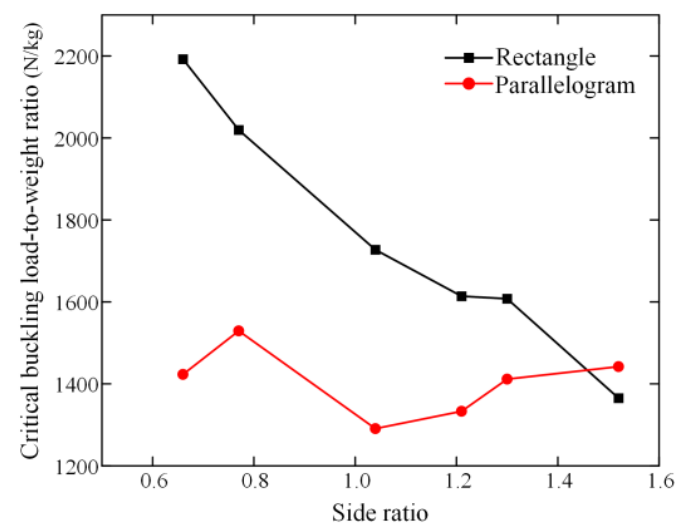

Figure 15. Critical buckling load-to-weight ratio vs. side ratio

We find that when the side ratio $l_{a} / l_{b}<1$ and the other parameters are constant, the radial stiffness-to-weight ratio of the rectangle waterbomb structure increases with the increase of $l_{a} / l_{b}$, and when $l_{a} / l_{b}>1$, the radial stiffness-to-weight ratio of the rectangle waterbomb structure decreases as $l_{a} / l_{b}$ increases. But regardless of whether $l_{a} / l_{b}>1$ or $l_{a} / l_{b}<1$, the critical buckling load-to-weight ratio decreases monotonously with increasing $l_{a} / l_{b}$.

Comparing the parallelogram waterbomb structures P12-P17, we find that the ratio $\mathrm{la} / \mathrm{lb}$ is sensitive to the effects of the radial stiffness-to-weight ratio and the critical buckling load-to-weight ratio. When the same initial parameters are used, P14 performs the best. The reason is that the parameter matching of P14 is the best.

\subsection{Effect of tilt angle}

Six types of parallelogram waterbomb structure models (P1-P6) with different tilt angles are established and analyzed to study the effect of tilt angle on the structure. The specific parameters are shown in Table 4. With all other parameters remaining constant, P1-P6 are simulated. The numerical calculation results show the changes in the ratio of critical buckling load-to-weight and the radial stiffness-to-weight ratio, as shown in Figures 16 and 17, respectively. 


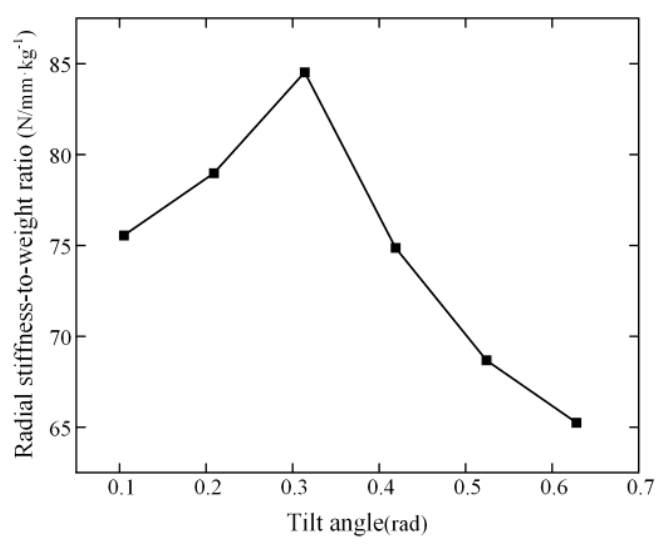

Figure 16. Radial stiffness-to-weight ratio vs. tilt angle

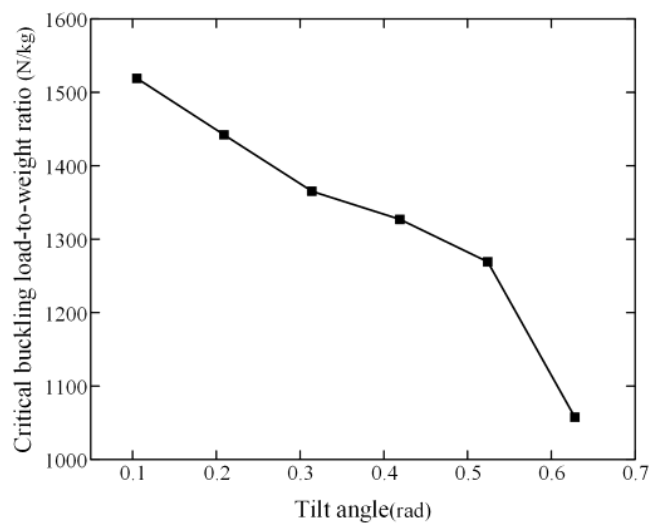

Figure 17. Critical buckling load-to-weight ratio vs. tilt angle

When all other initial variables remain constant, the critical buckling load-to-weight ratio decreases monotonically with the increase of the tilt angle $\alpha$, whereas the radial stiffness-to-weight ratio first increases and then decreases with the increase of $\alpha$. When $\alpha$ equals $0.314 \mathrm{rad}$, the radial stiffness-to-weight ratio reaches its maximum value. Because the critical buckling load is a measure to evaluate the difficulty of axial deformation of the waterbomb structure, and the radial stiffness is the best representation of the radial load bearing capacity of the waterbomb structure, in practical applications, we want the critical buckling load to be the smallest and the radial stiffness to be the greatest. It can be seen that after the other initial parameters are determined, the appropriate tilt angle $\alpha$ can be determined such that the mechanical properties of the waterbomb structure become optimal.

\subsection{Effect of gap in unit facet}

In order to study the effect of the gap in the unit facet on the waterbomb structures,

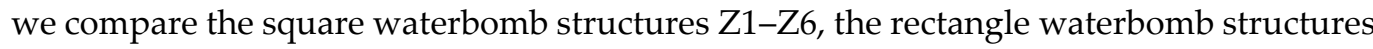
J3 and J7-J11, and the parallelogram waterbomb structures P3 and P7-P11. In total, 18 types of waterbomb structures are established and analyzed. The specific parameters of the models are shown in Tables 2, 3, and 4. With other variables kept constant, the numerical calculation results yield the changes in the critical buckling load-to-weight ratio and the radial stiffness-to-weight ratio with the increase of the cell gap, as shown in Figures 18 and 19 , respectively. 


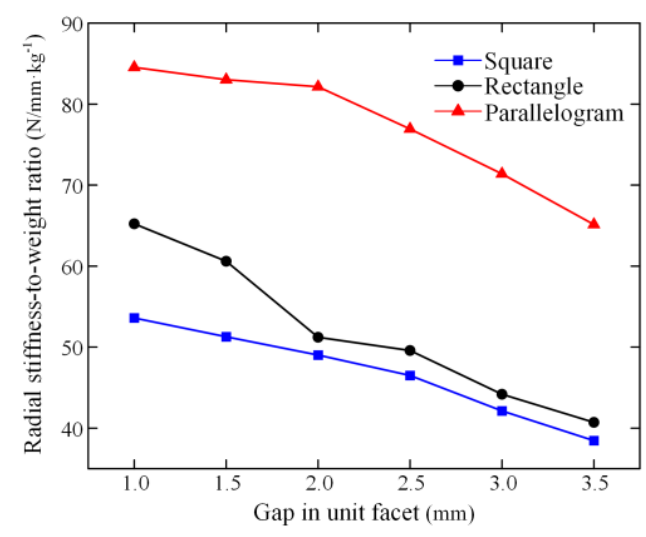

Figure 18. Radial stiffness-to-weight ratio vs. gap in unit facet

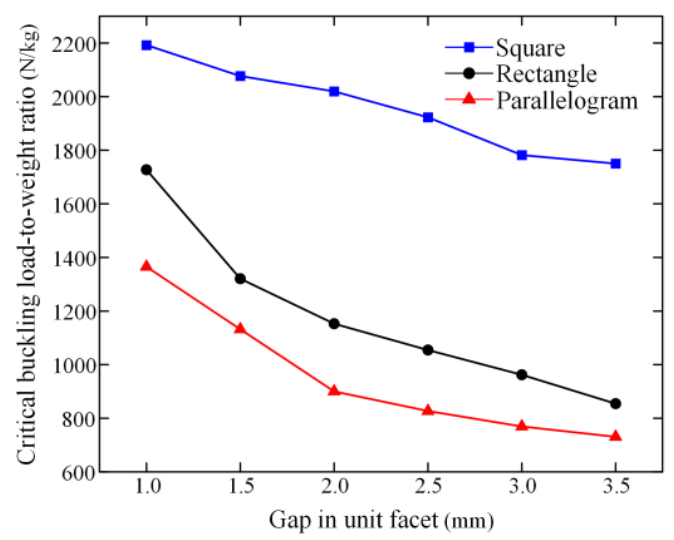

Figure 19. Critical buckling load-to-weight ratio vs. gap in unit facet

The comparison shows that as the gap in the unit facet, $v$, increases, the critical buckling load-to-weight ratios and the radial stiffness-to-weight ratios of the square, rectangle, and parallelogram waterbomb structures all show decreasing trends. The main reason is that as $v$ increases, the proportion of the flexible material used increases, which weakens the axial and radial rigidity of the waterbomb structures. These trends further imply the validity of the simulation results.

\section{Conclusions}

(1) Twist displacement mode along the axial direction is present in the parallelogram waterbomb structure under axial compression. By adjusting the parameters properly, the critical buckling load can be effectively reduced while the radial stiffness-to-weight ratio is significantly improved: compared with the conventional square waterbomb structure, the critical buckling load decreases by $55.4 \%$, and the radial stiffness-to-weight ratio increases by $67.6 \%$. In conclusion, the design method proposed in this paper can effectively improve the mechanical properties of waterbomb structures.

(2) When $l_{a} / l_{b}<1$ and the other parameters remain constant, the radial stiffness-toweight ratio of the rectangle waterbomb structure increases as $l_{a} / l_{b}$ increases. When $l_{a} / l_{b}>$ 1 , the radial stiffness-to-weight ratio of the rectangle waterbomb structure decreases as $l_{a} / l_{b}$ increases. Whether $l_{a} / l_{b}$ is $>1$ or $<1$, the critical buckling load decreases with $l_{a} / l_{b}$.

(3) For the parallelogram waterbomb structure, when other initial parameters remain constant, the critical buckling load decreases monotonously as the tilt angle $\alpha$ increases, whereas the radial stiffness-to-weight ratio first increases and then decreases as the tilt angle $\alpha$ increases. It can be concluded that with other initial parameters having been determined, the appropriate $\alpha$ can be determined through analysis to optimize the performance of the waterbomb structure. 
(4) As the gap in the unit facet, $v$, increases, the critical buckling loads and the radial stiffness-to-weight ratios of the square, rectangle, and parallelogram waterbomb structures all decrease.

Author Contributions: All authors have equally contributed to this article.

Funding: This work is supported by the National Natural Science Foundation of China (Nos. 51674134), Natural Science Foundation of Liaoning Province (No. 20180550167), Oversea Training Project of High Level Innovation Team of Liaoning (No. 2018LNGXGJWPY-ZD001). The authors would like to acknowledge the support of Collaborative Innovation Center for Coal Mine Robots.

Conflicts of Interest: The authors declare no conflict of interest.

\section{References}

1. Li, S.; Stampfli, J. J.; Xu, H. J.; Malkin, E.; Diaz, E. V.; Rus, D.; Wood, R. J. A Vacuum-driven Origami “Magic-ball” Soft Gripper. In Proceedings of the 2019 International Conference on Robotics and Automation (ICRA), IEEE, Montreal, QC, Canada, 74017408 May 2019.

2. Robertson, M.A.; Kara, O.C.; Paik, J. Soft pneumatic actuator-driven origami-inspired modular robotic "pneumagami". The International Journal of Robotics Research (in press).

3. Lee, D.; Kim, S.; Kim, J.; Park, J.; Cho, K. Origami Wheel Transformer: A Variable-Diameter Wheel Drive Robot Using an Origami Structure. SOFT ROBOT. 2017, 4, 163-180.

4. Lee, J.-Y.; Kang, B. B.; Lee, D.-Y.; Baek, S.-M.; Kim, W.-B.; Choi, W.-Y.; Song, J.-R.; Joo, H.-J.; Park, D.; Cho, K.-J. Development of a Multi-functional Soft Robot (SNUMAX) and Performance in RoboSoft Grand Challenge. Frontiers in Robotics and AI. 2016, 3:63.

5. Fang, H.; Zhang, Y.; Wang, K.W. Origami-based earthworm-like locomotion robots. BIOINSPIR BIOMIM. 2017, 12, 65003.

6. Onal, C. D.; Wood, R. J.; Rus, D. An Origami-Inspired Approach to Worm Robots. IEEE/ASME Transactions on Mechatronics. 2013, 18, 430-438.

7. Le, P. H.; Wang, Z.; Hirai, S. Origami structure toward floating aerial robot. In Proceedings of the 2015 IEEE International Conference on Advanced Intelligent Mechatronics (AIM), IEEE, Busan, South Korea, 1565-1569 July 2015.

8. Feng, Y.; Li, K.; Gao, Y.; Qiu, H.; Liu, J. Design and Optimization of Origami-Inspired Orthopyramid-Like Core Panel for Load Damping. Appl. Sci. 2019, 9, 4619.

9. Feng, H.; Ma, J.; Chen, Y.; You, Z. Twist of Tubular Mechanical Metamaterials Based on Waterbomb Origami. SCI. REP-UK. 2018, 8, 9522 .

10. Mukhopadhyay, T.; Ma, J.; Feng, H.; Hou, D.; Gattas, J. M.; Chen, Y.; You, Z. Programmable stiffness and shape modulation in origami materials: Emergence of a distant actuation feature. Appl. Mater. Today. 2020, 19, 100537.

11. Gillman, A.; Fuchi, K.; Buskohl, P. Truss-based nonlinear mechanical analysis for origami structures exhibiting bifurcation and limit point instabilities. Int. J. Solids Struct. 2018, 147, 80-93.

12. Fonseca, L.M.; Rodrigues, G.V.; Savi, M.A.; Paiva, A. Nonlinear dynamics of an origami wheel with shape memory alloy actuators. Chaos Soliton Fract. 2019, 122, 245-261.

13. Hanna, B.H.; Lund, J.M.; Lang, R.J.; Magleby, S.P.; Howell, L.L. Waterbomb base: a symmetric single-vertex bistable origami mechanism. SMART. MATER. STRUCT. 2014, 23, 94009.

14. Jiayao M.; Huijuan F.; Yan C.; Degao H.; Zhong Y. Folding of Tubular Waterbomb. Research. 2020, 1735081.

15. Bowen, L.; Springsteen, K.; Feldstein, H.; Frecker, M.; Simpson, T. W.; von Lockette, P. Development and Validation of a Dynamic Model of Magneto-Active Elastomer Actuation of the Origami Waterbomb Base. J. Mech. Robot. 2015, 7, 011010.

16. Glugla, D. J.; Alim, M. D.; Byars, K. D.; Nair, D. P.; Bowman, C. N.; Maute, K. K.; McLeod, R. R. Rigid Origami via Optical Programming and Deferred Self-Folding of a Two-Stage Photo-polymer. ACS. Appl. Mater. Interfaces. 2016, 8, 29658-29667.

17. Filipov, E. T.; Tachi, T.; Paulino, G. H. Origami Tubes Assembled into Stiff, yet Reconfigurable Structures and Metamaterials. Proc. Natl. Acad. Sci. U. S. A. 2015, 112, 12321-12326.

18. Berger J.; Wadley H.; McMeeking R. Mechanical metamaterials at the theoretical limit of isotropic elastic stiffness. Nature. 2017, 54, 533-537.

19. Bertoldi, K.; Vitelli, V.; Christensen, J.; van Hecke, M. Flexible mechanical metamaterials. Nat. Rev. Mater. 2017, 2, 1-11.

20. Ma, J.; Song, J.; Chen, Y. An origami-inspired structure with graded stiffness. Int J Mech Sci. 2018, 136, 134-142.

21. Wang, H.; Zhao, D.; Jin, Y.; Wang, M.; You, Z.; Yu, G. Study of collapsed deformation and energy absorption of polymeric origami-based tubes with viscoelasticity. Thin Wall. Struct., 2019, 144, 106246.

22. Zhai, Z.; Wang, Y.; Jiang, H. Origami-inspired, on-demand deployable and collapsible mechanical metamaterials with tunable stiffness. Proc. Natl. Acad. Sci. U. S. A. 2018, 115, 2032-2037.

23. Martinez, R.V.; Fish, C.R.; Chen, X; Whitesides, G.M. Elastomeric origami: programmable paper-elastomer composites as pneumatic actuators. Adv. Funct. Mater. 2012, 22, 1376-1384.

24. Filipov E. T.; Liu K.; Tachi T.; Schenk M.; Paulino G H. Bar and hinge models for scalable analysis of origami. Int J Solids Struct. 2017, 124, 26-45.

25. Guo, Z.; Gattas, J.; Wang, S.; Li, L.; Albermani, F. Experimental and numerical investigation of bulging behaviour of hyperelastic textured tubes. Int. J. Mech. Sci. 2016, 115, 665-675.

26. Ma, J.; You, Z. Energy Absorption of Thin-Walled Square Tubes With a Prefolded Origami Pattern - Part I: Geometry and Numerical Simulation. Int. J. Appl. Mech. 2013, 81, 011003. 
27. Holmes, D. Elasticity and Stability of Shape Changing Structures. Curr. Opin. Colloid. Interface Sci. 2019, 40, 118-137.

28. Lee, T.-U.; Yang, X.; Ma, J.; Chen, Y.; Gattas, J. M. Elastic buckling shape control of thin-walled cylinder using pre-embedded curved-crease origami patterns. Int. J. Mech. Sci. 2019, 151, 322-330.

29. Wang, C. M.; Zhang, Y. Y.; Ramesh, S. S.; Kitipornchai, S. Buckling analysis of micro- and nano-rods/tubes based on nonlocal Timoshenko beam theory. J. Phys. D. Appl. Phys. 2006, 39, 3904-3909.

30. Neves, M.M.; Rodrigues, H; Guedes, J.M. Generalized topology design of structures with a buckling load criterion. Structural Optimization. 1995. 10, 71-78.

31. Jafari Mehrabadi, S.; Sobhani Aragh, B.; Khoshkhahesh, V.; Taherpour, A. Mechanical buckling of nanocomposite rectangular plate reinforced by aligned and straight single-walled carbon nanotubes. Compos. Part B-Eng. 2012. 43, $2031-2040$.

32. Wu, C.-P.; Chen, Y.-J. A nonlocal continuum mechanics-based asymptotic theory for the buckling analysis of SWCNTs embedded in an elastic medium subjected to combined hydrostatic pressure and axial compression. Mech. Mater. 2020.148, 103514.

33. Panedpojaman, P.; Thepchatri, T.; Limkatanyu, S. Elastic buckling of cellular columns under axial compression. Thin Wall. Struct. 2019, 145, 106434.

34. Wagner, H. N. R.; Hühne, C.; Elishakoff, I. Probabilistic and deterministic lower-bound design benchmarks for cylindrical shells under axial compression. Thin Wall. Struct. 2020, 146, 106451.

35. Yuan, L.; Shi, H.; Ma, J.; You, Z. Quasi-static impact of origami crash boxes with various profiles. Thin Wall. Struct. 2019, 141, 435-446. 